\title{
Análisis de la Gestión de un Dispositivo Didáctico por un Estudiante de Profesorado en Matemática
}

\author{
Analysis of the Didactic Device Management by a Mathematics Teacher \\ Trainee
}

\author{
Ana Rosa Corica* \\ ORCID iD 0000-0002-3583-6081 \\ María Rita Otero** \\ ORCID iD 0000-0002-1682-9142
}

\begin{abstract}
Resumen
En este trabajo describimos resultados de la gestión de un dispositivo didáctico realizada por un estudiante del profesorado en matemática. El estudiante participó de un curso diseñado para la formación didácticomatemática, en el cual se aboga por un paradigma emergente basado en la investigación. Este adopta una concepción epistemológica de la matemática, que intenta poner en evidencia su utilidad y la vinculación con otras disciplinas. El estudiante llevó a cabo su implementación en un curso de quinto año de la escuela secundaria, en Argentina. La propuesta didáctica gestionada por el estudiante de profesorado posibilitó la realización de algunos gestos propios del paradigma de la investigación en las condiciones actuales de la escuela secundaria.
\end{abstract}

Palabras clave: Formación de profesores. Matemática. Teoría Antropológica de lo Didáctico. Enseñanza por investigación. Escuela secundaria.

\begin{abstract}
This work is set in the problematic of mathematics trainees. We describe the result of the didactic device management by a mathematics trainee. The student did a course that we designed to the didactic mathematic formation, which adopts a didactic non-traditional model based in research and the link with other disciplines. This student carried out his implementation in a fifth year of an Argentine high school. The didactic device enables the management of some gesture typical of the research and world questions paradigm, in the current condition of the high school.
\end{abstract}

Keywords: Teacher Training. Mathematics. Anthropological Theory of the Didactical. High School.

\footnotetext{
* Doctora en Ciencias de la Educación, Universidad Nacional de Córdoba (UNC); Investigador Adjunto del CONICET, investigador del NIECyT, Profesor Adjunto de la Facultad de Ciencias Exactas de la UNCPBA,Tandil, Buenos Aires, Argentina. Dirección postal: NIECyT - Facultad de Ciencias Exactas, Pinto 399, Tandil, Buenos Aires, Argentina, C.P: 7000. E-mail: acorica@exa.unicen.edu.ar.

${ }^{* *}$ Doctora en Enseñanza de las Ciencias, Universidad de Burgos (UBU); Investigador Principal del CONICET, investigador del NIECyT, Profesor Titular de la Facultad de Ciencias Exactas de la UNCPBA, Tandil, Buenos Aires, Argentina. Dirección postal: NIECyT - Facultad de Ciencias Exactas, Pinto 399, Tandil, Buenos Aires, Argentina, C.P: 7000. E-mail: rotero@exa.unicen.edu.ar.
} 


\section{Introducción}

La formación de profesores constituye una problemática abierta para la investigación en didáctica de la matemática. Las investigaciones, principalmente, se ubican en cuestionar el conocimiento matemático y didáctico que debe poseer el profesor para ejercer su profesión. Diversos investigadores acuerdan que la formación de profesores requiere de saber matemática con amplio domino del campo, y de una formación didáctica acorde a las exigencias institucionales y a la formación de los estudiantes como ciudadanos de hoy (ARTAUD; CIRADE; JULLIEN， 2011; BALL， 2000; CARDEÑOSO; FLORES; AZCÁRATE, 2001; GIMÉNEZ-RODRIGUEZ et al., 2009; PARADA; PLUVINAGE, 2014; ROBERT; POUYANNE, 2005; SHULMAN, 1987).

Aunque a lo largo de los años aumentó el número de investigaciones vinculadas a la temática, no hay acuerdo en la literatura científica acerca de cuáles son las competencias, disposiciones, etcétera, que debería conocer el profesor para favorecer el aprendizaje de sus estudiantes (GODINO, 2009).

En particular, Ruiz et al. (2014) indican que no es posible resolver, de manera acabada, el problema del equipamiento praxeológico que requieren los estudiantes de profesorado en matemática. Las cuestiones a las que este equipamiento debe aportar respuesta varían, y a partir de la investigación didáctica y del desarrollo de la propia práctica docente, surgen nuevos recursos, nuevas conocimientos, nuevos desafíos y la necesidad de modificar y renovar las antiguas praxis.

Este trabajo forma parte una investigación más amplia donde estudiamos cómo la formación puede incidir sobre la relación del profesor de matemática con las nociones que tiene que enseñar. De esta manera, cambia el problema de caracterizar el conocimiento didáctico-matemático del profesor y la incidencia de éstos sobre las prácticas docentes y sobre el aprendizaje matemático de los alumnos, por el problema mucho más amplio.

Se trata de estudiar la ecología institucional de las organizaciones didácticas y matemáticas, es decir, las condiciones que posibilitan su génesis y desarrollo institucional. En esta perspectiva, diseñamos e implementamos un curso destinado a estudiantes de profesorado en matemática (CORICA; OTERO, 2015, 2016a, 2016b, 2016c, 2017). El curso se propone, entre otros objetivos, que los estudiantes del profesorado en Matemática (EPM) puedan involucrarse en un paradigma emergente basado en la investigación.

En este trabajo reportamos resultados vinculados a la gestión de un dispositivo 
didáctico (DD) por parte de un EPM que experimentó nuestro curso, y cuyo dispositivo permite realizar gestos propios de la pedagogía de la investigación. Además, nos proponemos responder las siguientes preguntas de investigación: ¿Qué condiciones y restricciones tiene que afrontar el EPM para gestionar el DD? ¿Qué gestos de la pedagogía de la investigación puede realizar la comunidad que el EPM dirige?

\section{Marco Teórico}

La Teoría Antropológica de lo Didáctico (CHEVALLARD, 1999, 2007, 2013a, 2017) propone introducir en los sistemas de enseñanza encuentros con el saber motivado por las necesidades del estudio. Se procura el análisis de preguntas fundamentales (en general, no matemáticas), que crean el currículum, y este se desarrolla mediante el estudio de las mismas, que a su vez producen nuevas preguntas y exterioriza las obras a emplear. En este paradigma prima el liderazgo de las cuestiones (y las respuestas que les corresponden), atenuando el liderazgo de las obras que la pedagogía tradicional suele asignarles. Esta propuesta se materializa en un DD denominado Recorrido de Estudio e Investigación (REI).

Un sistema didáctico $S(X, Y, Q)$, propio del paradigma de la investigación que involucra una enseñanza por REI, se caracteriza por un conjunto de alumnos $X$, que estudia una pregunta $Q$, con la ayuda de un profesor o un conjunto de profesores $Y$, con el propósito de aportar una posible respuesta $R$ a $Q$. La producción de $R$ requiere que el sistema didáctico $S$ disponga de instrumentos, recursos, obras.

Según la gestión que se realice de un REI, este puede ser: cerrado, si el profesor impone determinados recorridos que conduce a la comunidad de estudio a encontrarse con nociones matemáticas elegidas por el profesor; semi-abierto, si el profesor elige la cuestión por indagar de manera que el recorrido pase por cierta obra matemática; abierto, si el rol del profesor se ubica en imponer, de vez en cuando, la decisión de no ir a encontrar determinada obra, que le parece estar aún fuera del alcance de los estudiantes (CHEVALLARD, 2017).

Llevar adelante la metodología propia de una enseñanza por REI, requiere incorporar gestos didácticos, que implican modificaciones radicales con respecto a la enseñanza tradicional (CHEVALLARD, 2007, 2013b, 2013c). A continuación, describimos cuatro gestos que consideramos esenciales en la gestión de un REI: 
- La dialéctica del estudio y de la investigación. Una investigación supone la combinación del estudio de preguntas y respuestas: no es posible investigar sin estudiar y, a su vez, un estudio genuino es productor de preguntas a ser investigadas.

- La dialéctica del individuo y el colectivo. Los estudiantes con su director de estudio acuerdan el conjunto de tareas a realizar y negociar las responsabilidades que debe asumir cada uno.

- La dialéctica de entrar y salir de tema. Si la pregunta es amplia y generativa, es necesario habilitar la posibilidad de salir del tema, incluso hasta puede ser necesario salir de la disciplina de referencia, y reingresar posteriormente.

- La dialéctica de las cajas negras y las cajas claras. Se refiere a la necesidad de establecer si una obra merece ser estudiada, aclarada, analizada etc., o si ciertos saberes se dejarán en un nivel de gris. Quedarían en gris saberes que no son esenciales para responder la cuestión generatriz o sus cuestiones derivadas.

\section{Metodología}

Proponemos una metodología de investigación cualitativa de corte exploratoria, descriptiva e interpretativa (HERNÁNDEZ; FERNÁNDEZ; BAPTISTA, 2010). El estudio se desarrolló en un curso de quinto año de la escuela secundaria argentina, dirigido por un EPM. Este gestionó el estudio de un DD que permite realizar algunos gestos propios de la pedagogía de la investigación.

El DD se inicia con el análisis de una situación que se ubica en comparar planes de telefonía celular, analizando el estado real de los planes que ofrecen diferentes compañías (RODRÍGUEZ; HIDALGO; SIERRA, 2013; CORICA, 2016). La implementación del DD requirió de once sesiones (dieciséis horas). Para cada sesión de la implementación, el investigador registró el audio general, realizó notas de campo y recogió las producciones escritas de los estudiantes.

Para el análisis de la gestión del DD se elaboró un Modelo Praxeológico de Referencia (MPR). Pues, para interpretar el proceso de estudio es necesario disponer de un punto de vista epistemológico (CHEVALLARD, 2013c). El MPR constituye una manera de interpretar las praxeologías por estudiar; se trata de una propuesta provisional a contrastar experimentalmente y, por lo tanto, susceptible de ser modificado y revisado. 


\subsection{Características del estudiante de profesorado}

El EPM era un estudiante de cuarto año de una carrera de profesorado en matemática de una Universidad Nacional Argentina, que experimentó nuestro curso para la formación didáctico-matemática (CORICA; OTERO, 2016a, 2016b, 2017). El objetivo del curso es que los estudiantes del profesorado adopten un modelo didáctico basado en la investigación y en la vinculación de la matemática con otras disciplinas. Se trata de un EPM que carece de experiencia docente, y conoce la escuela secundaria por su vivencia como alumno de la institución, y por lo estudiado en su proceso formativo.

\subsection{Características del curso en el que se desarrolló la investigación}

La implementación se desarrolló en un curso de quinto año, en una escuela secundaria pública de gestión privada en Argentina. En el curso se destinan tres horas semanales para estudiar matemática, segmentadas en un encuentro de dos horas y un encuentro de una hora. El curso estaba compuesto por $\mathrm{N}=35$ estudiantes cuyas edades oscilaban entre 15 y 16 años.

El grupo estaba habituado a una enseñanza tradicional de la matemática y en las demás disciplinas, pero los estudiantes mostraron interés en participar y explicar sus propuestas, más allá de que las técnicas empleadas fueran adecuadas o no. El EPM participó como ayudante del profesor del curso durante dos meses antes de implementar su DD. Esto le permitió conocer al grupo, la forma en que estudiaban y contribuyó a que los estudiantes interactuaran con quien en poco tiempo sería su profesor.

\section{Modelo praxeológico de referencia}

A continuación, presentamos las características esenciales de un MPR en torno al estudio de la cuestión $Q_{0}$ : En el mercado existen diversas compañías de telefonía celular y queremos contratar una, ¿Por cuál optar? ¿Por qué?

En la Figura 1 hay un esquema donde se propone una arborescencia de posibles cuestiones que se derivan de $Q_{0}$. Se pueden profundizar detalles de esta construcción en CORICA (2016). 


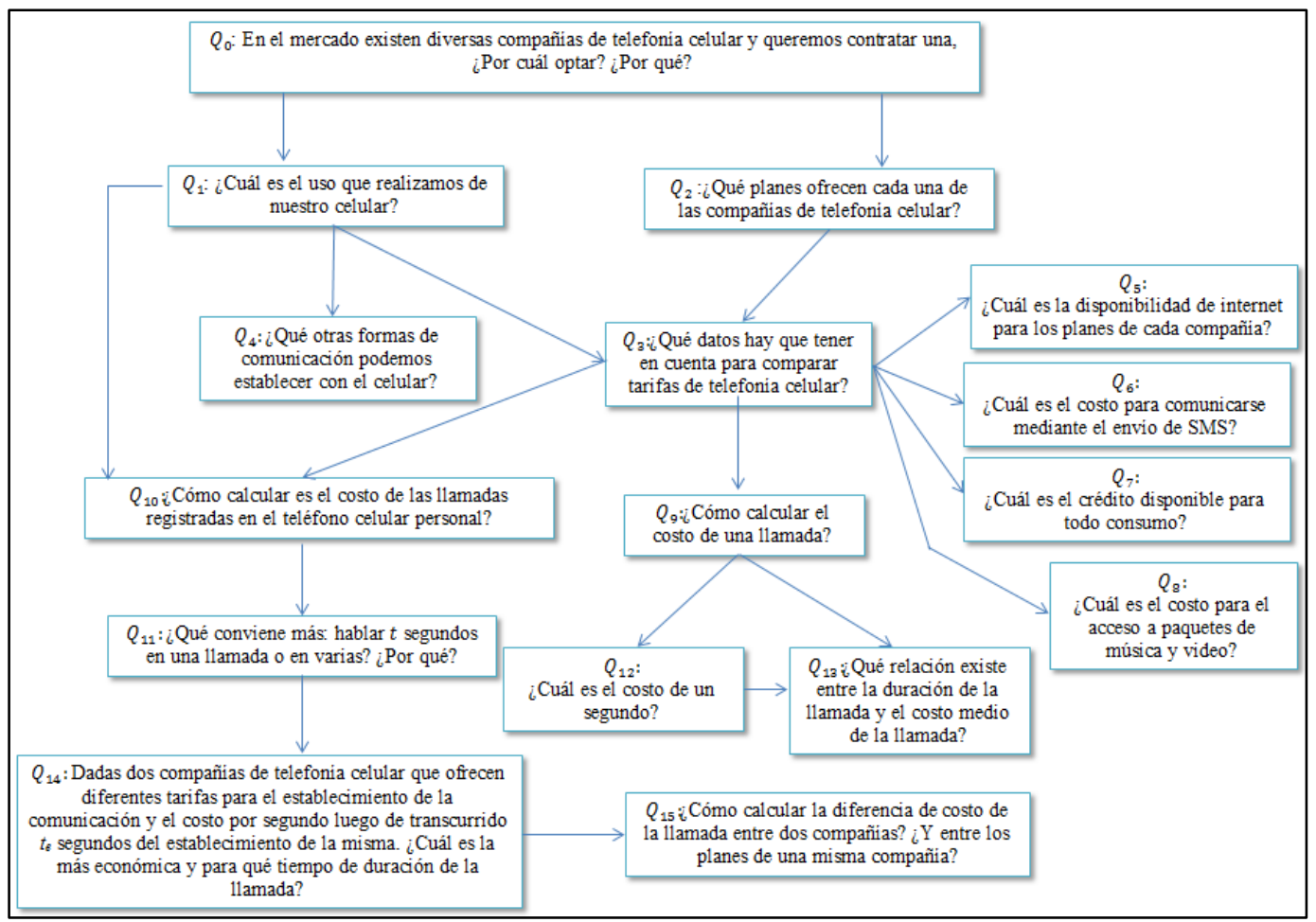

Figura 1 - Modelo praxeológico de Referencia Fuente: Elaboración propia

En el MPR pueden tratarse, entre otras, las quince cuestiones siguientes. $Q_{1}:{ }_{\zeta}$ Cuál es el uso que realizamos de nuestro celular? y $Q_{4}:$ ¿Qué otras formas de comunicación podemos establecer con el celular? tienen como propósito indagar acerca del empleo de la compañía de telefonía celular que realiza cada cliente y definir cuáles son sus necesidades. Esto resulta ser de vital importancia al momento de decidir qué compañía de telefonía contratar.

Por otro lado, $Q_{2}$ : ¿Qué planes ofrecen cada una de las compañías de telefonía celular? tiene como objetivo indagar acerca de las características de los diferentes planes que ofrece cada compañía. Esta exploración deriva en el estudio de las siguientes cuestiones: $Q_{3}$ : ¿Qué datos hay que tener en cuenta para comparar planes de telefonía celular? Esto último nos introduce a preguntarnos: ¡donde queda la questión 4 Q ${ }_{4}$ COMPROBAR se la numeración está correcta! $Q_{5}$ : ¿Cuál es la disponibilidad de internet para los planes de cada compañía?, $Q_{6}$ : ¿Cuál es el costo para comunicarse mediante el envío de $S M S ?, Q_{7}:$ ¿Cuál es el crédito disponible para todo consumo?, $Q_{8}:$ ¿Cuál es el costo para el acceso de paquetes de música y video?, $Q_{9}:$ ¿Cómo calcular el costo de una llamada?

Las preguntas $Q_{5}, Q_{7}$ y $Q_{8}$, son preguntas que se pueden responder rápidamente, revisando la información brindada por cada compañía. Sin embargo, las preguntas $Q_{6}$ y $Q_{9}$ son las que requieren mayor profundización en relación a la respuesta que se obtenga de $Q_{7}$, tornando problemática la situación inicial. La duración de las comunicaciones mediante 
llamadas y la cantidad de SMS que se pueden enviar dependen de los minutos y SMS libres indicados por las compañías y al crédito disponible para todo consumo.

En particular, del estudio de $Q_{9}$, se derivan las cuestiones: $Q_{10}$ : ¿Cómo calcular el costo de las llamadas registradas en el teléfono celular personal?, $Q_{11}:$ ¿Qué conviene más: hablar t segundos en una llamada o en varias? ¿Por qué?, $Q_{12}:$ ¿Cuál es el costo de un segundo?, $Q_{13}$ : ¿Qué relación existe entre la duración de la llamada y el costo medio de la llamada?, $Q_{14}$ : Dadas dos compañías de telefonía celular que ofrecen diferentes tarifas para el establecimiento de la comunicación y el costo por segundo luego de transcurrido $t$ segundos del establecimiento de la misma. ¿Cuál es la más económica y para qué tiempo de duración de la llamada?, $Q_{15}$ ¿ ¿Cómo calcular la diferencia de costo de la llamada entre dos compañías? ¿Y entre los planes de una misma compañía?

Las posibles respuestas a las cuestiones derivadas de $Q_{0}$, requieren recorrer praxeologías matemáticas que, en el diseño curricular de la escuela secundaria, aparecen poco articuladas o se encuentran ausentes. Estas praxelogías son: estadística descriptiva, función constante, función lineal, función racional, función definida a trozos, función techo, función piso, función módulo, estimación por redondeo y truncamiento, ecuaciones e inecuaciones lineales, sistema de ecuaciones lineales, dominio continuo y discreto, función continua y discontinua, límite de funciones en el infinito. El estudio de $Q_{0}$ permite reencontrar la utilidad de las distintas praxeologías matemáticas y vincularlas para estudiar una situación actual.

\section{Análisis la gestión del dispositivo didáctico dirigido por el EPM}

En este apartado describimos las características esenciales del estudio realizado por la comunidad en torno a la cuestión $Q_{0}$ : En el mercado existen diversas compañías de telefonía celular y queremos contratar una, ¿Por cuál optar? ¿Por qué? Describimos las situaciones que se estudiaron en las sucesivas sesiones, enfatizando el análisis en el tipo de preguntas que realiza el EPM y los estudiantes. Pues, uno de los gestos fundamentales en una enseñanza por investigación es la dialéctica del estudio e investigación, y la formulación de preguntas es esencial para que sea transitada durante el estudio.

El dispositivo didáctico que dirigió el EPM se inició con la pregunta: q0: ¿De qué compañía de telefonía celular son usuarios? ¿Por qué la eligieron? Esto dio origen a que los estudiantes aportaron respuestas inmediatas, caracterizadas por indicar la compañía de la que son usuarios. Se obtuvieron respuestas como las siguientes: 
$\mathrm{A}_{1}$ : Papá tiene Claro.

$\mathrm{A}_{2}$ : Tengo el plan que toda la familia tiene. Hablo gratis con todos.

$\mathrm{A}_{3}$ : Claro es más barato.

$\mathrm{A}_{4}$ : Antes tenía Claro, pero donde vivo no tiene señal entonces tengo Personal.

$\mathrm{A}_{5}$ : CLARO. Con CLARO puedo llamar gratis.

(Diálogo entre profesor y estudiantes, 2016).

Las respuestas ponen en evidencia que los estudiantes no se encuentran informados acerca de los planes de las compañías de las que son usuarios. La elección de la compañía es atribuida a una herencia familiar, o a decisiones poco fundamentadas. Solo $\mathrm{A}_{4}$ indica por qué cambió de compañía. En cambio, A5 pone en evidencia su poca información acerca de los planes que ofrecen otras compañías de la que no es usuario. Ante esta respuesta, el EPM intervino indicando que las compañía Movistar y Personal también ofrecen la posibilidad de comunicarse mediante llamadas sin costo, a usuarios que comparten la misma compañía.

Como la discusión no avanzaba y se obtenían respuestas inmediatas, no tornándose problemática la situación inicial, el EPM propuso las siguientes preguntas: $q_{1}$ : Para lo que usan el plan, ¿les sirve? $q_{2}:$ ¿Qué empresa nos conviene contratar si las tres tienen similar promoción?

La pregunta $q_{1}$ no fue explorada, solo se obtuvieron respuestas afirmativas por parte de los estudiantes. No se procedió a registrar y analizar datos del empleo que realiza cada usuario de los planes que son clientes. Esto hizo que no se recorrieran las preguntas $Q_{1}$ y $Q_{4}$, que se proponen en el MPR. Estas se refieren a explorar en profundidad el empleo que realiza cada usuario del teléfono celular y las posibilidades de uso que tendría según el plan contratado. Esto permitiría recurrir a praxeologías de estadística para poder aportar respuesta. Estas nociones no forman parte del diseño curricular de quinto año y el EPM no contaba con la disposición temporal necesaria para incidir en que el estudio prosiga en este sentido.

Para continuar con el análisis de $q_{2}$, el EPM proporcionó a los alumnos información disponible al momento de realizar el estudio, sobre los diferentes planes de abono, de las tres compañías de telefonía celular que tienen a los estudiantes como usuarios. Esta información fue una ayuda al estudio necesaria para que los alumnos ingresen a la problemática de la situación inicial.

Hubiera sido deseable que los estudiantes buscaran esta información en internet, explorándolas y la organizándolas, pues esto les permitiría ingresar a una de las tareas básicas de la estadística que es organizar, sintetizar y presentar información, pero por restricciones temporales esto no fue posible. En general, los planes de las compañías de telefonía celular, contienen una amplia información acerca de costo de abonos, y para cada uno de ellos: megas 
disponibles para el uso de internet, números telefónicos para hablar de manera ilimitada, crédito disponible para todo consumo, SMS incluidos en el abono, costo para re-compra de internet, forma y costo de la tasación para llamada local y de larga distancia, forma y costo de la tasación para llamada local y de larga distancia, luego de agotar el crédito disponible, acceso a paquetes de música y video, minutos disponibles para hablar incluidos en el abono.

Los estudiantes se dispusieron a analizar la información de los planes, en grupos compuestos por dos o tres integrantes. Cada grupo discutió la información y formuló nuevas preguntas para dar respuesta a $q_{2}$. Las cuestiones fueron compartidas y discutidas por toda la comunidad. De este modo, se acordó estudiar las siguientes preguntas redactadas por los alumnos y que identificamos con la nomenclatura $p_{x}$ :

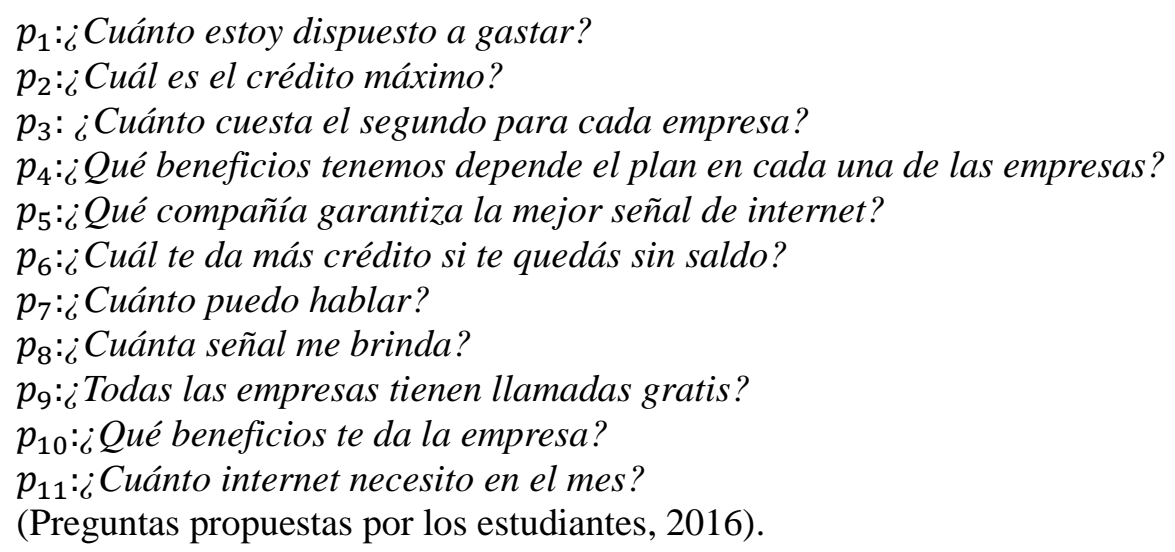

Estas preguntas integraron el medio didáctico inicial en el que se fundamentó el estudio y cuya constitución fue de los estudiantes. Esta primera tarea de acordar qué preguntas estudiar, generó la revisión de la propuesta de cada grupo y la manera de formular las preguntas, dando origen, también, a nuevas preguntas. De esta manera, los estudiantes comenzaron a transitar un aspecto esencial de la pedagogía de la investigación que es la formulación de preguntas.

También, la dialéctica del individuo y el colectivo ocupó un lugar central para poder definir el medio didáctico. Si bien, el rol del profesor fue fundamental para tornar problemática la situación, el estudio de la información de los planes de las compañías y las preguntas generadas por los alumnos hizo que se definiera un medio didáctico rico en el conjunto de preguntas a estudiar. Así también, durante el estudio surgieron nuevas preguntas por parte de los estudiantes (en ocasiones implícitas en su hacer, que destacamos por su relevancia $\left.\left(p_{x, y}\right)\right)$ y por parte del profesor que surgen como ayudas necesarias al estudio para posibilitar la continuidad del análisis (estas preguntas las identificamos como $q_{z}$ ).

A continuación, los estudiantes y el profesor analizaron qué preguntas admitían 
respuesta inmediata y cuáles no serían estudiadas. Pues, uno de los aspectos que señalan los alumnos por qué elegir una compañía de telefonía celular es la calidad de la señal $\left(p_{5} \mathrm{y} p_{8}\right)$. Se acordó que las preguntas $p_{5}$ y $p_{8}$ no serían estudiadas, porque la comunidad de estudio no contaba con suficientes herramientas para poder hacerlo. Abordar estas preguntas involucra, también, alejarse del foco central del EPM, que es estudiar la utilidad de la Matemática para responder $q_{0}$. Sin embargo, mediante el empleo de Internet es posible conocer el área de cobertura de las compañías a partir del análisis de un mapa interactivo (por ejemplo en: www.claro.com.ar/empresas/institucional/cobertura/).

Las preguntas $p_{1}$ y $p_{11}$ resultan ser personales. La respuesta depende de las decisiones y uso que establezca realizar el usuario. Las preguntas $p_{4}, p_{6}, p_{9}$ y $p_{10}$ admiten respuesta inmediata al analizar el plan que ofrece cada compañía. Finalmente, el grupo acordó profundizar el estudio en $p_{2}, p_{3}$ y $p_{7}$. Si bien, $p_{2}$ parecería ser una pregunta que admite respuesta inmediata a partir del análisis del crédito disponible para todo consumo que ofrecen los planes, su análisis se torna problemático al ser estudiada en conjunto con $p_{3}$ y $p_{7}$.

A continuación, el EPM propuso que cada grupo se ocupara de estudiar los planes de una compañía, atendiendo a que toda la comunidad aborde las tres compañías consideradas. En principio, los estudiantes acordaron estudiar las siguientes preguntas de manera conjunta, pues establecer cuánto puedo hablar $\left(p_{7}\right)$ depende del crédito disponible que contempla el plan contratado $\left(p_{2}\right): p_{2}:$ ¿Cuál es el crédito máximo? $p_{7}: ¿$ Cuánto puedo hablar?

Elaborar una respuesta a estas preguntas requiere comprender de qué manera las compañías de telefonía celular tasan las comunicaciones mediante llamadas. Al revisar los términos y condiciones legales que propone cada compañía, se concluye en lo siguiente: el primer bloque de treinta segundos tiene un costo fijo, y luego de este período se comienza a tasar un costo por cada segundo que dure la comunicación. Por otro lado, la duración de las llamadas que se pueden efectuar se encuentra vinculada con el crédito disponible para todo consumo. Para maximizar el empleo de este crédito, es necesario establecer la comunicación y dejar que se agote el mismo. Si no sucede esto, cortar y volver a establecer la comunicación siempre se encuentra afectado por el costo del establecimiento de la llamada.

Los estudiantes analizaron para cada compañía, según el crédito disponible, el máximo de segundos que se puede establecer una comunicación. Las primeras técnicas de los alumnos fueron aritméticas. Por ejemplo, en la Figura 2 se puede observar los cálculos propuestos por los estudiantes del Grupo 2, sobre el plan de la compañía Claro.

Estos restaron al crédito disponible, el costo de una llamada de treinta segundos, y 
luego calcularon para ese resultado, el tiempo de duración de una llamada. Inferimos que los estudiantes consideran el costo de una llamada sin interrupción. Es decir, una llamada ideal en la que se inicia la comunicación y se continúa hasta que se agota el crédito.

Por otro lado, se observa la deficiencia de los estudiantes en los cálculos propuestos. De manera arbitraria, reducen el costo de las llamadas en los primeros treinta segundos a dos decimales $(\$ 1,30)$, siendo que la empresa factura $\$ 1,3050$. Proceden del mismo modo para el costo por segundo luego de transcurrido treinta segundos de iniciada la comunicación: los alumnos consideran un costo de $\$ 0,04$ el segundo, siendo que la compañía establece un costo de $\$ 0,0435$. Esto hace que al calcular la máxima duración de la comunicación con el crédito disponible, tenga una diferencia alrededor de 108 segundos con relación a lo calculado por los estudiantes.

Por otro lado, el cálculo del tiempo máximo en el que se podría mantener la comunicación es indicado mediante decimales. No se identifica que la forma de tasación de la compañía es por segundo, es decir, la fracción de segundo es perdida por el usuario. Esto fue habitual en las resoluciones de los estudiantes y requirió de intervenciones del EPM para que estos interpreten los resultados obtenidos en el contexto de la situación (Figura 2).

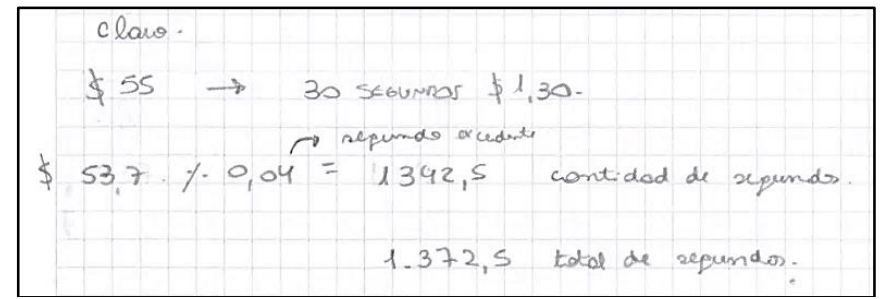

Figura 2 - Resolución del Grupo 2

Fuente: Protocolo del Grupo 2

Los estudiantes del Grupo 11 calcularon el costo de una llamada que consume todo el crédito disponible sin interrupción (Figura 3). Los cálculos se efectuaron para los tres planes que ofrece una misma compañía. Este grupo realizó una adecuada interpretación de resultados considerando solo la parte entera de sus cálculos.

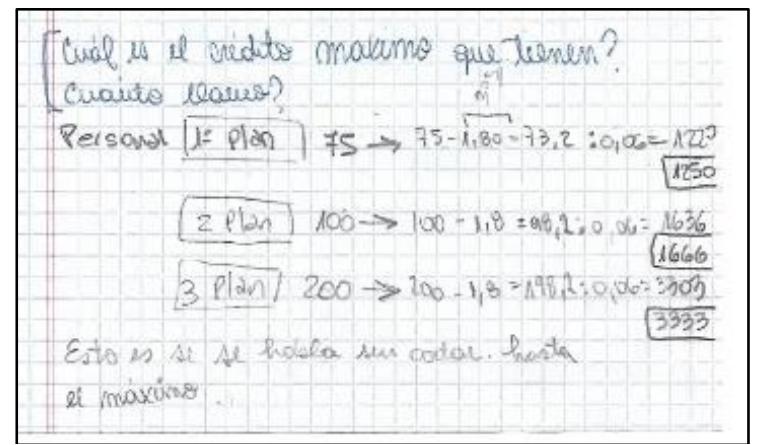

Figura 3 - Resolución del Grupo 11

Fuente: Protocolo del Grupo 11 
En la Figura 4 se indica el protocolo del Grupo 6. Se observa una inadecuada interpretación de cómo calcular el tiempo máximo de duración de una llamada. Los estudiantes calcularon el costo de una llamada de un minuto y luego calcularon cuántas llamadas de un minuto se pueden realizar con el crédito disponible. Las técnicas empleadas permiten concluir que se pueden realizar veinte llamadas de un minuto con el crédito disponible. Este resultado que responde a la pregunta implícita en el seno del grupo: $p_{2,1}$ : ¿Cuántas llamadas de t minutos se pueden realizar? Esta se aleja del foco central del estudio conjunto de $p_{2}$ y $p_{7}$ y no permite maximizar el empleo del crédito disponible.

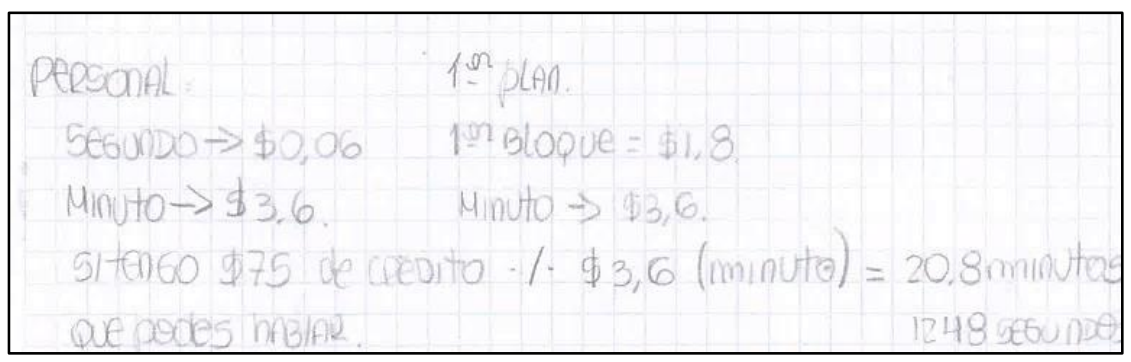

Figura 4 - Resolución del Grupo 6

Fuente: Protocolo del Grupo 6

En la Figura 5 se indica la producción de los estudiantes del Grupo 8. Los alumnos calculan el costo de una llamada de once minutos. Como el caso del Grupo 6, los estudiantes se alejan del foco central de $p_{2}$ y $p_{7}$, respondiendo con su hacer a la pregunta implícita en el seno del grupo $p_{2,2}:$ ¿Cuál es el costo de una llamada de t minutos de duración?

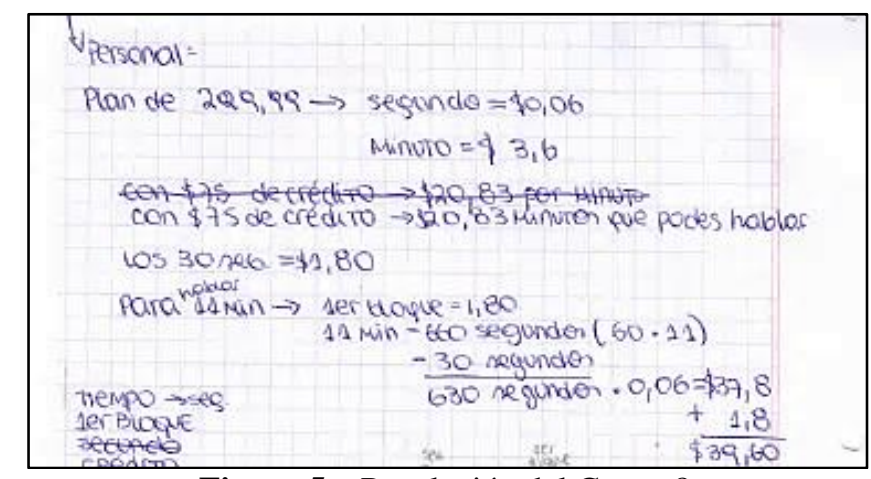

Figura 5 - Resolución del Grupo 8

Fuente: Protocolo del Grupo 8

A partir de las respuestas acordadas por los diferentes grupos para $p_{2}$, se concluyó en la tabla que se indica a continuación. Esta fue confeccionada en el pizarrón por el EPM (Tabla 1).

Tabla 1 - Tiempo máximo de llamadas según la compañía

\begin{tabular}{|c|c|c|c|c|c|}
\hline \multicolumn{2}{|r|}{ Claro } & \multicolumn{2}{|c|}{ Movistar } & \multicolumn{2}{|c|}{ Personal } \\
\hline$\$ 55$ & $1260,54 \mathrm{seg}$ & $\$ 50$ & $732,04 \mathrm{seg}$ & $\$ 75$ & $\begin{array}{l}1250 \mathrm{seg} \\
1248\end{array}$ \\
\hline
\end{tabular}

Fuente: Respuesta de los estudiantes 
En la Tabla 1, se indica el crédito disponible para todo consumo que establece cada compañía para el plan más económico, y los segundos como máximo que puede durar una llamada que se inicia y continua hasta que se agota el crédito disponible para todo consumo. En particular, los estudiantes necesitaron de las ayudas de estudio ofrecidas por el EPM para poder comprender los resultados que fueron obteniendo durante sus cálculos y poder interpretar qué preguntas estaban respondiendo y qué respuestas se corresponden con $p_{2}$. Así por ejemplo, con el propósito de comprender la forma de tasación de llamadas que realizan las compañías de telefonía celular e indagar a cerca de los valores obtenidos en la Tabla 1, el EPM realizó la siguiente pregunta:

EPM: ¿Cuál es el crédito de Movistar? ¿Se puede hablar hasta 732, 46 segundos?

$\mathrm{A}_{8}$ : Cuando llegás a 732 se te corta el plan. Si tenés liberado te cobra en el otro mes, sino se te corta.

(Diálogo entre profesor y estudiantes, 2016).

De esta manera, se revisaron las respuestas recogidas en la Tabla 1. Por otro lado, para profundizar en la forma de tasación de llamadas, el EPM indicó: Si ustedes realizan una llamada, ¿cómo cobra el sistema? $\left[q_{3}\right]$. Como no se registraron respuestas de los estudiantes, el EPM propuso discutir las siguientes situaciones:

EPM: Hablaron 3,8 segundos, ¿cuál es el costo de esta llamada?

$\mathrm{A}_{6}$ : Hasta 30 segundos te cobran ese precio establecido. Te cobran 30 segundos [...]

EPM: [...] si hablo 40,1 segundos...

$\mathrm{A}_{7}$ : Te cobran 41 segundos.

(Diálogo entre profesor y estudiantes, 2016).

Esta conversación, que se estableció entre el EPM y todo el grupo, dio lugar a que el profesor introdujera al medio la definición de función techo y función piso, como entorno tecnológico que justifica las técnicas empleadas.

El EPM propuso salir del tema para poder profundizar en tareas referidas a función techo y función piso. Estas indagan las técnicas necesarias para dar respuesta a $p_{2}$. Son tareas matemáticas puntuales que no habían sido estudiadas por los alumnos en otras oportunidades. A continuación, se indican los tipos de tarea al que refieren: $T_{1}$ : Calcular la función piso en un punto, $T_{2}$ : Calcular la función techo en un punto.

Finalizado este estudio, el EPM propuso a los alumnos que realizaran el análisis de $p_{7}$ : ¿Cuánto puedo hablar? Esto demandó acordar la necesidad de establecer una expresión que permita calcular el tiempo que se puede hablar según el crédito disponible, y que sea utilizada para cualquier compañía. Los estudiantes propusieron expresiones como la siguiente (Figura 6): 


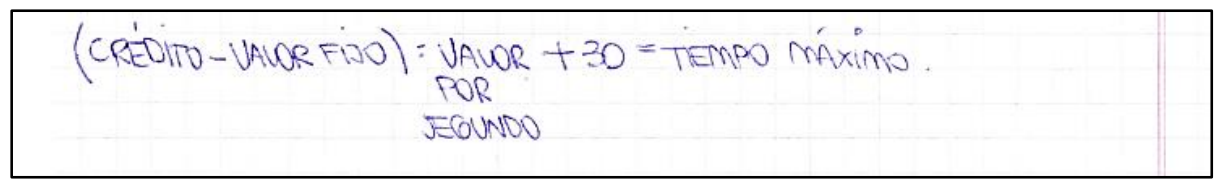

Figura 6 - Resolución del Grupo 9

Fuente: Protocolo del Grupo 9

Las fórmulas de los estudiantes se encontraban compuestas por diferente nomenclatura, según sus propias decisiones. Para unificar criterio, la comunidad de estudio acordó utilizar la siguiente nomenclatura: Crédito $(c)$, Precio por segundo $(P s)$, Primer bloque $(P B)$. De esta manera, las fórmulas propuestas por los diferentes grupos, resultó ser: $\frac{c-P B}{P S}+$ 30. En esta expresión, no se considera la situación real estudiada con anterioridad. Esto requirió que el EPM indagara a los estudiantes acerca de qué ocurre si al realizar los cálculos resulta un número con decimales. Los estudiantes recuperaron el entorno tecnológico introducido al medio didáctico por el EPM y propusieron la siguiente expresión: $\left[\frac{c-P B}{P S}+30\right\rfloor$.

A continuación, el EPM solicitó retomar el estudio de $p_{2,2}:{ }_{¿}$ Cuál es el costo de una llamada de t minutos de duración?, que surgió en el hacer de algunos de los grupos.

Los estudiantes propusieron expresiones como las siguientes (Figuras 7 y 8):

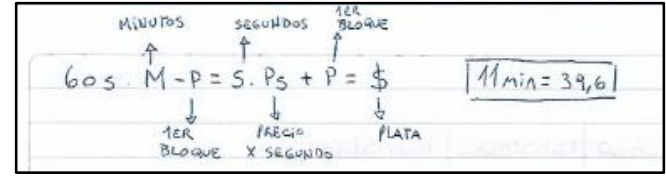

Figura 7 - Producción del Grupo 7

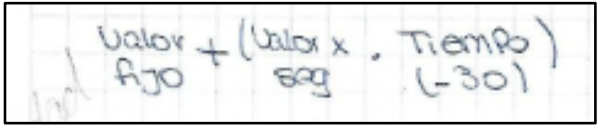

Figura 8 - Producción del Grupo 8 Fuente: Protocolo del Grupo 8

Estas expresiones fueron registradas en el pizarrón y discutidas con todo el grupo, para poder establecer cuál de ellas modela la situación. Se acordó que la expresión que corresponde es la propuesta por el Grupo 2. Esta expresión resulta incompleta porque no considera la forma de tasación de las llamadas. Esto hizo que el EPM interviniera, solicitando el costo de llamadas cuyo tiempo de duración es menor a treinta segundos y estudiando si esta situación se ajusta al modelo propuesto. Así también, el estudio requirió de intervenciones del EPM para poder definir el domino de validez de la expresión indicada. Finalmente, la comunidad de estudio concluyó en la expresión que se indica en el protocolo del Grupo 7 (Figura 9).

$$
\begin{aligned}
& P_{B}=\text { Primeros } 30 \text { sejundos } \\
& \int P_{B} \quad 1 \leq T \leq 30 \\
& \left\{\begin{array}{l}
P B \\
P_{B}+(P S(T-30)) \\
30<T<\left\lfloor\frac{C-P B}{P S}+30\right] \rightarrow F U N C i O ́ N \text { A TROZOS }
\end{array}\right.
\end{aligned}
$$

Figura 9 - Producción del Grupo 7 Fuente: Protocolo del Grupo 7 
En particular, para $p_{2,2}$ se cuestiona el costo de la llamada para $t$ en minutos. Aquí se acordó considerar $t$ en segundos, por la forma de tasación de las llamadas de las compañías.

A continuación, el profesor propuso introducir la definición de función definida a trozos, siendo que no había sido estudiada con anterioridad por los alumnos. También propuso tareas que involucran la noción de continuidad de funciones, pues esta emerge del hacer de tareas vinculadas con función definida a trozos.

Las tareas propuestas se sintetizan en los siguientes tipos de tarea: $T_{3}$ : Establecer el dominio de validez de expresiones definidas a trozos; $T_{4}$ : Establecer la imagen de funciones definidas a trozos; $T_{5}$ : Hallar puntos de intersección de funciones definidas a trozos con los ejes de coordenadas; $T_{6}$ : Representar gráficamente funciones definidas a trozos; $T_{7}$ : Establecer intervalos de positividad y negatividad de funciones definidas a trozos; $T_{8}$ : Hallar la expresión de funciones definidas a trozos representadas gráficamente; $T_{9}$ : Establecer funciones continuas y discontinuas.

A continuación, el profesor propuso representar, de manera gráfica, las funciones propuestas para cada compañía de telefonía celular. En particular, el estudio matemático de las funciones a trozos consideradas se centró en el análisis de funciones definidas a trozos discontinuas en uno o dos puntos, pero en cada intervalo que define la rama de las funciones el dominio es continuo.

Sin embargo, la función costo tiene dominio discreto para cada intervalo del dominio que definen sus ramas. De esta manera, la comunidad de estudio se sumergió en la problemática de cómo trazar la gráfica de $c(t)\left[q_{4}\right]$. Los estudiantes decidieron qué técnicas emplear para representar la función. Por ejemplo, el Grupo 2 realizó la gráfica mediante el empleo de GeoGebra ${ }^{\circledR}$ y mediante técnicas de lápiz y papel (Figura 10).

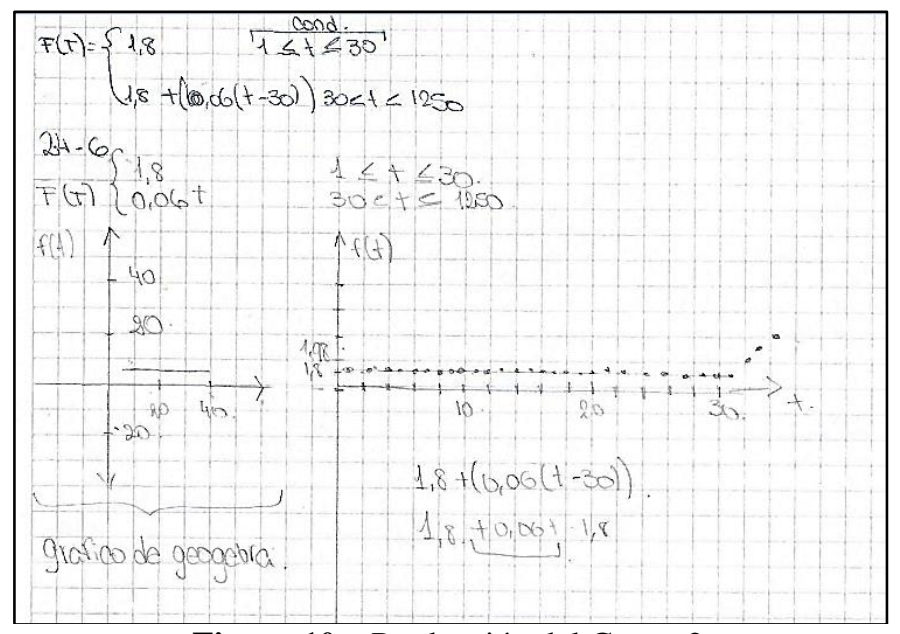

Figura 10 - Producción del Grupo 2 Fuente: Protocolo del Grupo 2 
Los estudiantes propusieron dos gráficas (Figura 10). El gráfico de la izquierda es copia de lo realizado con GeoGebra ${ }^{\circledR}$, y por la escala empleada se puede interpretar que la gráfica $c(t)$ es una recta paralela al eje de abscisas. Mientras que el gráfico de la derecha fue realizado con lápiz y papel. En este caso, los estudiantes dan indicios de interpretar el dominio que corresponde a la situación e indican puntos en vez de una función continua.

Algunos estudiantes no evidenciaron comprender el dominio de la función. Por ejemplo, en el protocolo del Grupo 3 (Figura 11), los estudiantes construyeron la gráfica de la función costo para la compañía Claro. La técnica empleada para construir la gráfica se basa en la representación de la función en una tabla. Esta es construida de manera adecuada, siendo que se consideran valores de tiempo naturales, pero al trazar la gráfica, la representación resulta ser una función continua.

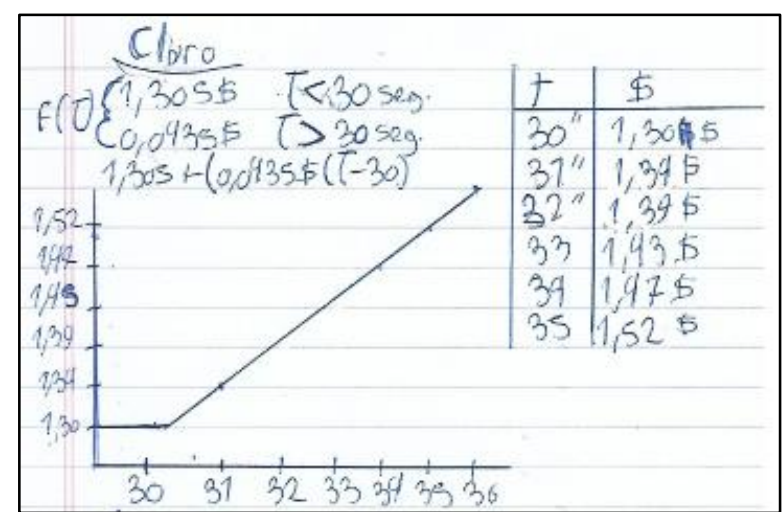

Figura 11 - Producción del Grupo 3

Fuente: Protocolo del Grupo 3

El EMP propuso discutir la propuesta de cada grupo y el estudio prosiguió a que los alumnos realicen la gráfica para los planes de las tres compañías y analicen los resultados obtenidos en estudios anteriores durante la construcción del modelo. Esta tarea resultó ser relevante, pues permitió que los estudiantes comprendan el comportamiento de funciones con dominio discreto, y de qué manera las compañías tasan las comunicaciones.

A continuación, se retomó el estudio de la cuestión: $p_{3}$ : ¿Cuánto cuesta el segundo para cada empresa? La comunidad de estudio aportó las siguientes respuestas:

A8: Depende del plan.

A9: Un segundo es igual a 0,0435.

EPM: ¿Pero si hablo 10 segundos?

$\mathrm{A}_{10}$ : Lo que sale 30 segundos.

EPM: Pero me dicen lo que sale la llamada, ¿pero cuánto el segundo?

¿Cuánto 10 segundos?

$\mathrm{A}_{13}$ : Tenés que hacer 1,305 dividido 10 igual a 0,1305

$\mathrm{A}_{15}$ : Si lo multiplicás por 30 no te da.

EPM: ¿Te sale más caro o barato?

$\mathrm{A}_{17}$ : Cuanto más hablás, te sale menos caro el segundo.

(Diálogo entre profesor y estudiantes, 2016). 
En principio, los estudiantes consideraban que el costo del segundo es el indicado por las compañías en la sección de términos y condiciones, haciendo de $p_{3}$ una pregunta que admite respuesta inmediata. Los estudiantes no lograban ingresar en la problemática de $p_{3}$, por lo que las intervenciones del EPM resultaron ser necesarias ayudas al estudio, para que estos comprendieran cuál es el costo del segundo y de qué manera depende según el tiempo de duración de la comunicación. Con el objetivo de profundizar el estudio, el EPM solicitó a los estudiantes que determinen una expresión que permita calcular el costo del segundo según la duración de la llamada. Los alumnos obtuvieron expresiones como la siguiente (Figura 12)

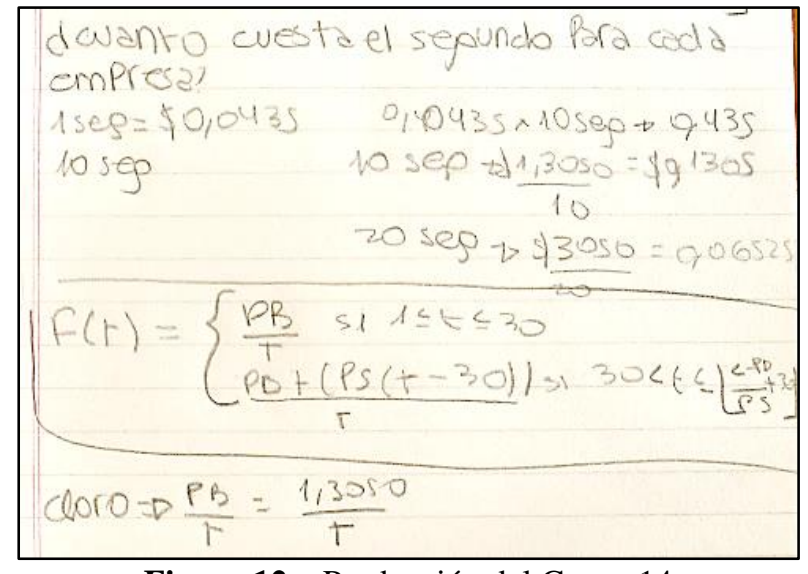

Figura 12 - Producción del Grupo 14 Fuente: Protocolo del Grupo 14

Siendo que las ramas de la función costo medio se compone de funciones racionales, el EPM definió este tipo de función porque no había sido estudiado por los alumnos.

Con el propósito de continuar analizando la función relacionada al costo medio, el EPM preguntó: ¿Qué ocurre cuando aumenta el tiempo? $\left[q_{5}\right]$ Los estudiantes analizaron la expresión de la función, pudiendo concluir que cuando aumenta la duración de la llamada, el costo del segundo disminuye. Como las respuestas obtenidas resultaban incompletas, el EPM propuso estudiar el comportamiento de la función $f(x)=\frac{0,1350}{x}$ que corresponde a la primera rama de la función costo medio de la compañía Claro.

En primer lugar, el EPM propuso analizar, de manera descontextualizada, la función, realizando la gráfica de la misma mediante el estudio de tablas de valores. Algunos estudiantes realizaron la gráfica mediante Geogebra ${ }^{\circledR}$ y otros mediante lápiz y papel. Este estudio descontextualizado permitió que los alumnos comprendan el comportamiento de la función para luego retomar el análisis de la función costo medio.

Finalmente, el EPM propuso estudiar tareas vinculadas al análisis de funciones racionales. Estas hacen referencia a los siguientes tipos de tarea: $T_{10}$ : Establecer el dominio de validez de expresiones racionales; $T_{11}$ : Establecer la imagen de funciones racionales; $T_{12}$ : 
Hallar puntos de intersección de funciones racionales con los ejes de coordenadas; $T_{13}$ : Representar gráficamente funciones racionales; $T_{14}$ : Establecer intervalos de positividad y negatividad de funciones racionales; $T_{15}$ : Hallar la expresión de funciones racionales representadas gráficamente.

El estudio se concluyó con el análisis de la función racional involucrada en la función costo medio. Si se profundiza en el análisis, el estudio comprendería nociones de límite de funciones, como indicamos en el MPR. Estas nociones se proponen estudiar en sexto año de la escuela secundaria argentina. Por estas restricciones, el EPM concluyó el análisis de la función costo medio en esta instancia.

Durante el desarrollo de diez sesiones el EPM gestionó el estudio de $q_{0}$ y las cuestiones derivadas. En varias oportunidades el EPM tuvo la necesidad de distanciar el estudio del eje central de $q_{0}$, y profundizar en el análisis de nociones matemática, para aportar respuesta a las preguntas: $p_{2}, p_{3}$ y $p_{7}$ y a sus derivadas $\left(p_{2,1}, p_{2,2}, q_{3}, q_{4}, q_{5}\right)$. Por esta razón, se decidió que en la última sesión los estudiantes aborden una situación que recupere el estudio de $q_{0}$ y aporten respuesta habiendo emprendido una vasta exploración de los planes que ofrecen las tres compañías. A continuación indicamos la situación final (Figura 13).

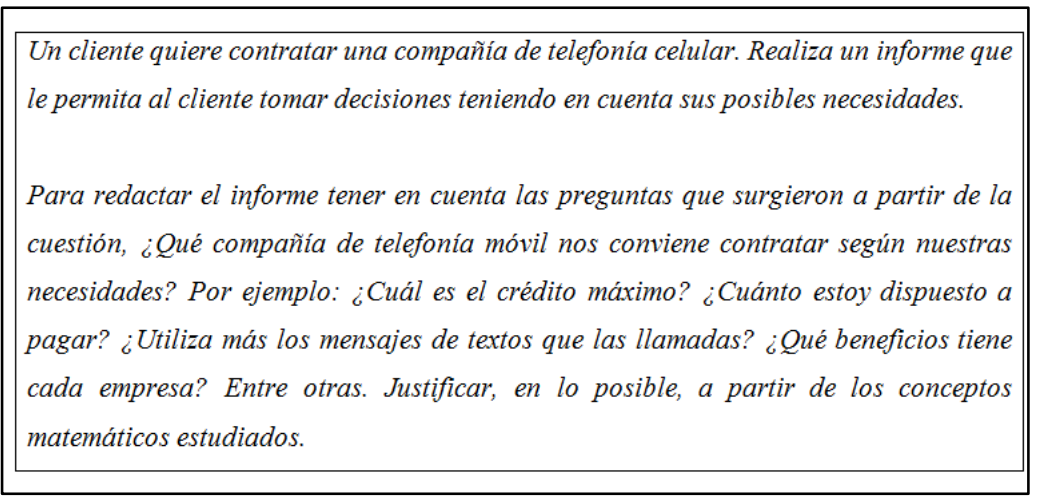

Figura 13 - Situación final

Fuente: Elaboración del investigador

Esta última tarea generó que la comunidad de estudio realizara conjeturas de posibles clientes y formulara posibles decisiones a tomar. La propuesta de los alumnos recuperó lo realizado desde el inicio del estudio, profundizando en la utilidad de la matemática para el análisis de la situación.

\section{Conclusiones}

El trabajo describe resultados de la implementación de un DD por un EPM en clases habituales de matemática, de una escuela secundaria en Argentina. El DD tiene la 
particularidad de permitir realizar gestos propios de la pedagogía de la investigación. El propósito de la investigación es responder las siguientes preguntas: ¿Qué condiciones y restricciones tiene que afrontar el EPM para gestionar el DD? ¿Qué gestos de la pedagogía de la investigación puede realizar la comunidad que el EPM dirige?

La situación inicial $q_{0}$ generó gran interés en el grupo de estudiantes. Lo atribuimos a que remite a una situación actual y de interés social. El estudio fue rico en el conjunto de preguntas derivadas, transitando de esta manera uno de los gestos fundamentales de la pedagogía de la investigación: la dialéctica del estudio e investigación.

La formulación de preguntas es de vital importancia en este tipo de estudio y el EPM logró que el grupo formulara y estudiara las propias. Las ayudas al estudio ofrecidas por el EPM fueron fundamentales para avanzar y sostener la problematización de la situación inicial. Los estudiantes de la escuela secundaria que participaron de la investigación están habituados a una enseñanza tradicional e instantánea de la matemática, donde las tareas tienen respuestas únicas e inmediatas. Esta es una de las condiciones y restricciones fundamentales que tuvo que afrontar el EPM para gestionar el estudio.

A pesar de que el análisis de las preguntas derivadas de $q_{0}$ fue regulado por el EMP, los alumnos asumieron la responsabilidad de búsqueda de respuesta, proponiendo y estudiando situaciones concretas. Las respuestas fueron validadas por la propia experiencia y conocimientos de los estudiantes y por la constatación entre los grupos de trabajo y el EPM. Esto último se corresponde con uno de los gestos propios de la pedagogía de la investigación: la dialéctica del colectivo y el individuo. Esta fue transitada de manera parcial, porque el medio de estudio si bien fue gestado a partir de la propuesta de cada pequeño grupo, los estudiantes compartieron las mismas respuestas, recorriendo las mismas praxeologías.

Durante el estudio dirigido por el EPM, la matemática surgió como una necesidad para dar respuesta a la situación inicial y condujo a reencontrar la funcionalidad de algunas praxeologías estudiadas por los alumnos en años anteriores tales como: función constante, función lineal, estimación por redondeo y truncamiento, ecuaciones e inecuaciones lineales, dominio continuo. El estudio también exigió que los alumnos recorrieran nuevas praxeologías para ellos, relativas a: función racional, función definida a trozos, función techo, función piso, dominio discreto, función continua y discontinua. En particular, las nociones: función definida a trozos, función techo y función piso, no son propuestas para ser estudiadas en el diseño curricular de la escuela secundaria.

Las praxeologías matemáticas recorridas durante el estudio no cubren totalmente las 
praxeologías que proyectamos recorrer en el MPR. Por restricciones institucionales, tales como el diseño curricular vigente y el espacio temporal cedido, el EPM no pudo ampliar el estudio de praxeologías necesarias para profundizar en el análisis. Estas praxeologías se refieren a estadística descriptiva y límite de funciones en el infinito.

Durante la dirección del estudio, se alternaron instancias más liberales y otras más arbitrarias. Las instancias liberales corresponden a los momentos de formulación de preguntas derivadas de $q_{0}$. Esto incluso cuando el EPM indicó cómo profundizar en el análisis de $q_{0} \mathrm{y}$ las cuestiones derivadas, aportando nuevas preguntas para profundizar en la exploración del estudio. Aquí, las decisiones tomadas fueron fruto de la deliberación colectiva, bajo la supervisión del profesor.

Mientras que el estudio se tornó de carácter arbitrario en las instancias donde el EPM requirió el estudio de tareas matemáticas puntuales. Fue quien decidió qué tareas puramente matemáticas estudiar sobre: función techo, función piso, función definida a trozos y función racional. Así también, con qué nivel de profundización de acuerdo a la formación previa de los estudiantes. Esto hizo que la dialéctica de entrar y salir del tema y la dialéctica de las cajas claras y negras fueran dirigidas totalmente por el EMP. Atribuimos esto a la inexperiencia docente del EMP, la restricción temporal, la necesidad de cumplir con un diseño curricular, las tareas proyectadas para el estudio propuestas por el profesor que cedió el espacio y el sistema evaluativo imperante en la institución, que exigió contar con una evaluación tradicional para acreditar a los estudiantes.

Nuestros trabajos continúan enfocándose en formar EPM en el estudio y gestión de dispositivos didácticos en los que la matemática recupere su funcionalidad. Si bien, el DD gestionado en este trabajo no es un REI abierto, permite transitar algunos de los gestos propios que involucra una enseñanza por investigación por EPM (quienes no son expertos en la TAD), en las condiciones actuales de la escuela secundaria Argentina.

\section{Referencias}

ARTAUD, M.; CIRADE, G.; JULLIEN, M. Intégration des PER dans l'equipement praxéologique du professeur. Le cas de la formation initiale. En: BOSCH, M. et al. (Ed.). Un panorama de la TAD. Barcelona: Centre de Recerca Matemàtica, 2011. p. 769-794.

BALL, D. Bridging practices: Intertwining content and pedagogy in teaching and learning to teach. Journal of Teacher Education, University of Michiganv, v. 51, n. 3, p. 241-247, may. 2000.

CARDEÑOSO, J.; FLORES, P.; AZCÁRATE, P. El desarrollo profesional de los profesores de matemáticas como campo de investigación en educación matemática. En: GÓMEZ P.; RICO L. (Ed.). Iniciación a la investigación en didáctica de la matemática. Homenaje al profesor Mauricio Castro. Granada: Universidad de Granada, 2001. p. 233-244. 
CHEVALLARD, Y. L'analyse des pratiques enseignantes en théorie anthropologique du didactique. Recherches en Didactique des Mathématiques, Grenoble, v. 19, n. 2, p. 221-266, 1999.

CHEVALLARD, Y. Passé et présent de la théorie anthropologique du didactique. En RUIZHIGUERAS, L., ESTEPA, A.; JAVIER GARCIA, F. (Ed.). Sociedad, Escuela y Mathemáticas. Aportaciones de la Teoría Antropológica de la Didáctico: Universidad de Jaén, 2007. p. 705-746.

CHEVALLARD, Y. Enseñar Matemáticas en la Sociedad de Mañana: Alegato a favor de un contraparadigma emergente. REDIMAT, Universidad de Barcelona. v. 2, n. 2, p.161-182, jun. 2013 a.

CHEVALLARD, Y. Journal du Seminaire TAD/IDD. Théorie Anthropologique du Didactique \& Ingénierie Didactique du Développement. 2013b. Disponible en: <http://yves.chevallard.free.fr/>. Acceso: 19 feb. 2018.

CHEVALLARD, Y. Éléments de didactique du développement durable. Leçon 3, 2013c. Disponible en: <http://yves.chevallard.free.fr/>. Acceso: 19 febr. 2018.

CHEVALLARD, Y. ¿Por qué enseñar matemáticas en secundaria? Una pregunta vital para los tiempos que se avecinan. La Gaceta de la RSME, Real Sociedad Matemática Española, v. 20, n. 1, p. 159169, mar. 2017.

CORICA, A. Enseñanza por Investigación en el marco de la Teoría Antropológica de lo Didáctico: Una propuesta para estudiar las funciones a trozos. 1. ed. Tandil: UNCPBA, 2016.

CORICA, A.; OTERO, M. The Mathematics Teacher's Profession: the Perspective of Future, for that you consider submitting to assessment. European Journal of Science and Mathematics Education, v. 3 , n. 2 , p. 145-158, abr. 2015.

CORICA, A.; OTERO, M. Estudio de dispositivos didácticos propuestos por futuros profesores de Matemática: un análisis desde la TAD. Perspectiva Educacional, v. 55, n. 2, p. 21-37, jun. 2016 a.

CORICA, A.; OTERO, M. Diseño e implementación de un curso para la formación de profesores en matemática: una Propuesta desde la TAD. Bolema, v. 30, n. 55, p. 763-785, ago. 2016b.

CORICA, A.; OTERO, M. Análisis de la implementación de un dispositivo didáctico enmarcado en el paradigma de la investigación desarrollado por un estudiante del profesorado en matemática. In: CONGRESO INTERNACIONAL DE ENSEÑANZA DE LAS CIENCIAS Y LA MATEMÁTICA, 2, ENCUENTRO NACIONAL DE ENSEÑANZA DE LA MATEMÁTICA, 3, 2016, Tandil. Actas... Tandil: UNCPBA, 2016c. p 253-258.

CORICA, A.; OTERO, M. Análisis de un dispositivo didáctico propuesto por futuros profesores de matemática formados en la TAD. AIEM, v. 12, p. 79-95, nov. 2017.

GIMÉNEZ-RODRÍGUEZ, J. et al. Competencias profesionales en el máster de profesorado de secundaria. Uno, Graó, v. 51, p. 9-18, jun. 2009.

GODINO, J. Categorías de Análisis de los Conocimientos del Profesor de Matemáticas. UNION, Federación Iberoamericana de Educación Matemática, v. 20, p. 13-31, dic. 2009.

HERNÁNDEZ, R.; FERNÁNDEZ, C; BAPTISTA, M. Metodología de la investigación. 5. ed. México: Mc Graw Hill, 2010.

PARADA, R.; PLUVINAGE, F. Reflexiones de profesores de matemáticas sobre aspectos relacionados con su pensamiento didáctico. Relime, CLAME, v. 17, n.1, p. 83-113, mar. 2014.

ROBERT, A.; POUYANNE, N. Formar formadores de maestros de matemáticas de educación media: ¿Por qué y cómo? Educación Matemática, SOMIDEM, v. 17, n. 2, p. 35-58, ago. 2005. 
RODRÍGUEZ, E.; HIDALGO, M.; SIERRA, T. La modelización a través de los recorridos de estudio e investigación: el caso de la comparación de tarifas de telefonía móvil. In: CONGRESO

INTERNACIONAL SOBRE LA TAD, 4., 2013, Toulouse. Actas... Toulouse: Université Toulouse, 2013. p. 421-452.

RUIZ, A. et al. Las Matemáticas para la Enseñanza en una Formación del Profesorado Basada en el Estudio de Cuestiones. Bolema, Universidade Estadual Paulista, v. 28, n. 48, p. 319-340, Abr. 2014.

SHULMAN, L. Knowledge and teaching: Foundations of the new reform. Harvard Educational Review, v. 57, n. 1, p. 1-22, feb. 1987.

Submetido em 20 de Fevereiro de 2018. Aprovado em 11 de Julho de 2018 